\title{
Mechanical and Dielectric Properties of InTe Crystals
}

\author{
Teena Mathew ${ }^{1}$, Ayyappacharuparambil Gopalanachary Kunjomana ${ }^{1 *}$, Keelapattu Munirathnam ${ }^{1}$, \\ Kunnath Appukuttan Chandrasekharan ${ }^{1}$, \\ Muthukrishnan Meena ${ }^{2}$, Chelliah Kamalakshiammal Mahadevan ${ }^{2}$ \\ ${ }^{1}$ Research Centre, Department of Physics, Christ University, Bangalore, India \\ ${ }^{2}$ Physics Research Centre, S. T. Hindu College, Nagercoil, India \\ Email: "kunjomana.ag@christuniversity.in
}

Received October 15, 2012; revised November 17, 2012; accepted November 26, 2012

\begin{abstract}
The mechanical properties of indium telluride (InTe) crystals grown by the Bridgman technique were investigated at room temperature using a Vickers hardness tester. The microhardness is observed to vary nonlinearly with the applied load, $10-100 \mathrm{~g}$. The cleaved ingots are found to have high value of microhardness $\left(222.44 \mathrm{~kg} / \mathrm{mm}^{2}\right.$ at a load of $\left.25 \mathrm{~g}\right)$, which reflects an appreciable degree of strength due to their covalent bonding and homogeneity. The studies revealed that the dislocations in the grown crystals offered a resistance to fresh dislocations due to interaction. At higher loads, plastic deformation induces by slip, exhibiting a decrease in hardness from the peak value. The dielectric constant and dielectric loss of indium telluride crystals were evaluated in the frequency range, $1 \mathrm{kHz}-1 \mathrm{MHz}$ for different temperatures $\left(35^{\circ} \mathrm{C}-140^{\circ} \mathrm{C}\right)$. The frequency dependence of $\mathrm{AC}$ conductivity was analyzed as a function of temperature. The effect of temperature and frequency on the dielectric response of InTe crystals has been assessed on their cleavage faces and the obtained results are discussed.
\end{abstract}

Keywords: Indium Telluride; Bridgman Technique; Microhardness; Dielectric Constant; Dielectric Loss; AC Conductivity

\section{Introduction}

Indium telluride (InTe), a prominent semiconducting IIIVI compound, finds potential application in the fabrication of switching devices and has been used in semiconductor hetero-structures $[1,2]$. Among all the mechanical properties, hardness is a key factor governing the quality of such structures. Hence, considerable literature [3-6] exists on the microhardness studies of compound semiconducting crystals. The anisotropy of mechanical properties is associated with structural defects, chemical bonding, plastic deformation and their tendency towards crack formation and cleavage. Kunjomana and Chandrasekharan [3] have carried out the microindentation analysis on the prism faces of GaTe whiskers. The effect of annealing on the microhardness of zone-melted $\mathrm{In}_{\mathrm{x}} \mathrm{Bi}_{2-\mathrm{x}} \mathrm{Te}_{3}(\mathrm{x}=0.1$ to $0.5 \mathrm{at} \% \mathrm{In})$ was studied by Pandya et al. [4]. The mechanical properties of pure and doped InP have also been investigated [5]. It is reported that impurity hardening is much more pronounced at high temperatures than at room temperature. Arivuoli et al. [6] have described the growth and microhardness studies of arsenic, antimony and bismuth chalcogenides. However,

${ }^{*}$ Corresponding author. the microindentation analysis of indium monotelluride crystals has not been reported so far.

Under controlled conditions, InTe crystallizes in a layer structure with the space group I $4 / \mathrm{mcm}$ as described by Chattopadhyay et al. [7]. There exists strong covalent bonding within the layer planes with weak van der Waals bonding perpendicular to them, resulting in easy cleavage. The studies on the dielectric behaviour of chalcogenide materials are advantageous for understanding their conduction mechanism and the origin of dielectric losses [8]. The AC conductivity and dielectric properties of $\mathrm{Sb}_{2} \mathrm{Te}_{3}$ thin films have been investigated in the frequency range, $0.4-100 \mathrm{kHz}$ as a function of temperature [9]. Hegab et al. [10] have evaluated the dielectric properties and frequency dependence of $\mathrm{AC}$ conductivity of amorphous $\mathrm{Ge}_{15} \mathrm{Se}_{60} \mathrm{X}_{25}(\mathrm{X}=\mathrm{As}$ or $\mathrm{Sn})$ thin films deposited by thermal evaporation. Bose and Purkayastha [11] have determined the dielectric constants of $\mathrm{In}_{2} \mathrm{Te}_{3}$ crystals grown by the Bridgman method. But, InTe, being a member of III-VI family, is less investigated, as far as its dielectric properties are concerned. In view of the above considerations, the present report aims to investigate the mechanical and dielectric properties of indium monotelluride crystals. 


\section{Experimental}

\subsection{Growth and Structural Analysis}

Stoichiometric InTe crystals were grown from melt by the Bridgman method, using a vertical single zone furnace. The high pure $(99.999 \%)$ indium and tellurium were filled in a precleaned quartz ampoule of length 80 $\mathrm{mm}$ and inner diameter $10 \mathrm{~mm}$, sealed under a vacuum of $\sim 10^{-6}$ mbar and synthesized using a muffle furnace. The temperature profile of the furnace was studied for performing the growth experiments. The compound was melted by raising the temperature above the melting point $\left(696^{\circ} \mathrm{C}\right)$ in a tapered ampoule for a period of $48 \mathrm{~h}$ and translated at a rate of $5 \mathrm{~mm} / \mathrm{h}$. The X-ray Powder Diffraction (XRD) data of the grown crystals were recorded with a Philips X'pert diffractometer, subjecting Cuk $\alpha(\lambda=1.5418 \AA)$ radiation. Energy Dispersive Analysis by X-rays (EDAX) was carried out to assess the chemical homogeneity of the grown samples.

\subsection{Mechanical Measurements}

The mechanical strength of a grown crystal plays an important role in investigating the quality of a crystalline surface for any desired application. It is essential to know about the dislocation motion and stress relationships involved in the crystal for studying the mechanical properties. Therefore, a Vickers projection microscope (MVH-I) was employed to perform the indentations on the cleavage planes of the grown InTe crystals. The diamond indenter is in the form of a square pyramid, opposite faces of which make an angle of $136^{\circ}$ with one another. Subsequent impressions were made after a time lapse of 30 min to allow for any elastic recovery. In order to avoid mutual influence of the indentations, the process was carried out at different sites such that the distance between consecutive indentation marks is greater than the diagonal length $(d)$. The microhardness was computed for various loads using the "Quantimet software" coupled with the tester.

\subsection{Dielectric Measurements}

The dielectric characteristics of InTe crystals were investigated by monitoring the capacitance $\left(C_{\text {crys }}\right)$ and dielectric loss factor $(\tan \delta$ ) using a LCR meter (AGILENT 4284A) for different frequencies, viz. $100 \mathrm{~Hz}, 1 \mathrm{kHz}, 100$ $\mathrm{kHz}$ and $1 \mathrm{MHz}$. A good conductive surface layer was prepared by coating the samples with silver paste. The temperature was increased up to $140^{\circ} \mathrm{C}$ and the electrical parameters were recorded while cooling. The geometrical dimensions of the crystals were measured using travelling microscope and screw gauge (least count $=0.01$ $\mathrm{mm})$.

\section{Results and Discussion}

X-ray powder diffraction analysis of the sample confirmed the formation of InTe with tetragonal crystal structure. The estimated cell parameters, $a=b=8.437 \AA$ and $c=7.139 \AA$ are found to be quite consistent with the JCPDS card 30 - 0636. The density of the grown crystals $\left(6.336 \mathrm{~g} / \mathrm{cm}^{3}\right)$ calculated from the powder diffraction data supports the material property reported in the literature $[12,13]$. The EDAX profile (Figure 1) revealed the ratio of atomic percentages of In and Te as 49.98: $50.02 \mathrm{at} \%$, which shows reasonable agreement with the standard value.

In order to study the mechanical properties of a material, it is desirable to examine optically flat surfaces, free from any microstructures or irregularities. The crystals have been carefully cleaved at liquid nitrogen temperature and the polished slices were subjected to indentation. The Vickers hardness number is computed using the formula [14],

$$
H v=1.8544 P / d^{2} \mathrm{~kg} / \mathrm{mm}^{2}
$$

where $P$ is the applied load in kilograms and $d$ is the mean diagonal length in millimeters. Figure 2 represents the results of microhardness measurements on the (001)

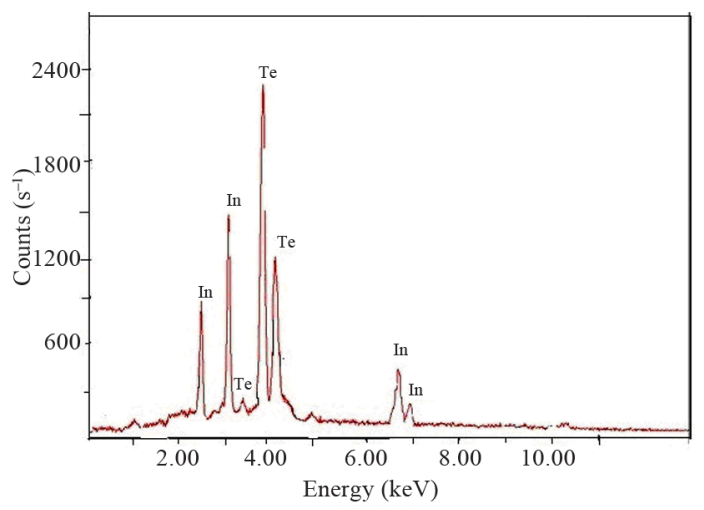

Figure 1. EDAX spectrum of InTe sample.

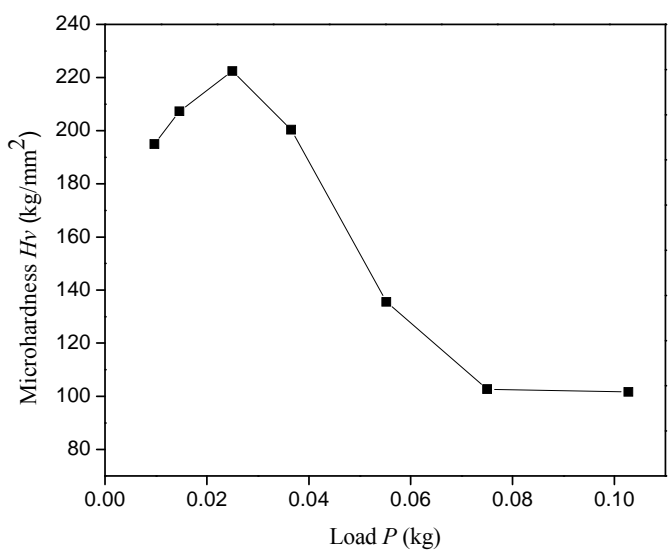

Figure 2. Plot of microhardness with load for InTe crystals. 
plane of InTe crystals. The applied load was varied from $10-100 \mathrm{~g}$, maintaining the dwell time at $15 \mathrm{~s}$ for all the samples.

The nonlinear behavior of hardness depends on internal and applied stress, work hardening and intrinsic plastic resistance of the material. The value of microhardness increases with increase in load and is found to be maximum $\left(222.44 \mathrm{~kg} / \mathrm{mm}^{2}\right)$ at $25 \mathrm{~g}$. This is attributed to the fact that one of the indium atoms has tetrahedral coordination with four tellurium atoms and exhibits $\mathrm{sp}^{3}$ hybridization [13]. Moreover, the presence of covalent bonding and the interaction between dislocations have a pronounced effect on the hardening mechanism. It attains a minimum value equal to $101.6 \mathrm{~kg} / \mathrm{mm}^{2}$ at a load of 100 $\mathrm{g}$. The decrease in hardness of InTe crystals is because of the gliding between the layers on the cleavage plane of InTe. Beyond $100 \mathrm{~g}$, the hardness remains constant, due to decrease in the resistance to the movement of dislocations. However, it is found to be greater than that of other class of semiconducting monotelluride compounds such as ZnTe $\left(82 \mathrm{~kg} / \mathrm{mm}^{2}\right)$, CdTe $\left(56 \mathrm{~kg} / \mathrm{mm}^{2}\right)$, CuTe $(19.2$ $\mathrm{kg} / \mathrm{mm}^{2}$ ) etc. [14]. Thus, a proper control on the growth conditions ensures quite an appreciable strength and quality of InTe crystals, which makes them suitable for the preparation of hetero-structures.

The study of dielectric behavior of chalcogenide semiconducting crystals reveals structural information, which helps to understand the conduction mechanism. Hence in the present work, the dielectric constant of the crystal was estimated in the frequency range $1 \mathrm{kHz}$ to 1 $\mathrm{MHz}$ by applying the relation [15],

$$
\varepsilon_{r}=\left[A_{\text {air }} / A_{\text {crys }}\right]\left[\left\{C_{\text {crys }}-C_{\text {air }}\left(1-A_{\text {crys }} / A_{\text {air }}\right)\right\} / C_{\text {air }}\right]
$$

where $A_{\text {crys }}$ is the area of the crystal touching the electrode and $A_{\text {air }}$ is the area of the electrode. Since the crystal area was smaller than the plate area of the cell, air capacitance $\left(C_{\text {air }}\right)$ was also measured [15]. Figure 3 shows the frequency dependence of dielectric constant of indium telluride crystals at different temperatures. The dielectric constant $\left(\varepsilon_{r}\right)$ decreases with increase in frequency and shows a steeper dependence at high frequency region. Similar results were reported on the dielectric properties of $\mathrm{Sb}_{2} \mathrm{Te}_{3}$ thin films [9]. At low frequencies, $\varepsilon_{r}$ depends on deformational (electronic and ionic) and relaxation (orientational and interfacial) polarization. When the frequency is increased, the dipoles will no longer be able to rotate rapidly and the oscillations begin to lag behind the field. As the frequency is further increased, the dipoles will be randomly aligned and the orientation is stopped. Hence, the dielectric constant decreases at higher frequency, approaching a constant value, corresponding only to the interfacial polarization.

The dependence of dielectric constant on temperature at various frequencies, $1 \mathrm{kHz}, 10 \mathrm{kHz}, 100 \mathrm{kHz}$ and 1 $\mathrm{MHz}$, is plotted in Figure 4. The dielectric constant increases with increase in temperature and this behavior becomes predominant at higher temperature and lower frequency. The increase in dielectric constant with temperature is due to the fact that, the orientational polarization is governed by the thermal motion of molecules. The dipoles do not orient at low temperature, but as the temperature increases, the orientation of dipoles is facilitated and thus increases the value of orientational polarization, which in turn increases $\varepsilon_{r}$ [16].

Figures 5 and $\mathbf{6}$ indicate the variation of dielectric loss with frequency and temperature respectively. It is found that, the dielectric loss decreases with frequency and increases with temperature. The origin of the dielectric losses is associated with the relaxation phenomena, which is divided into three parts: conduction losses, dipole losses and vibrational losses. As the temperature increases, conductivity as well as electrical conduction losses increase and hence the value of the dielectric loss $(\tan \delta)$ increases [9].

At high frequency, AC conductivity $\left(\sigma_{a c}\right)$ increases with frequency, according to the equation [16],

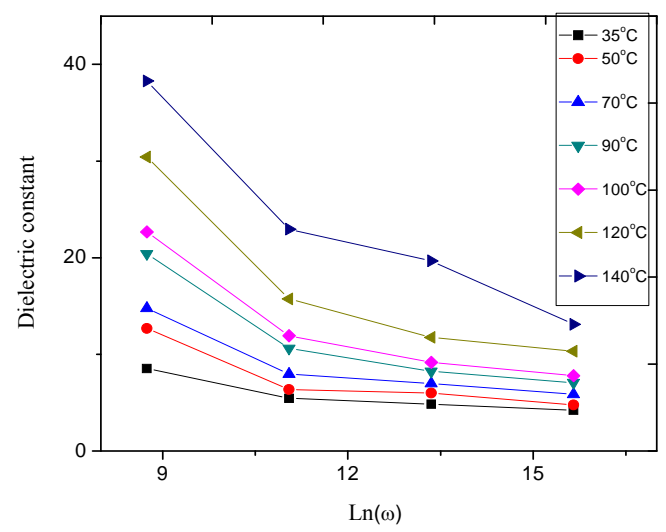

Figure 3. Frequency dependence of dielectric constant at different temperatures.

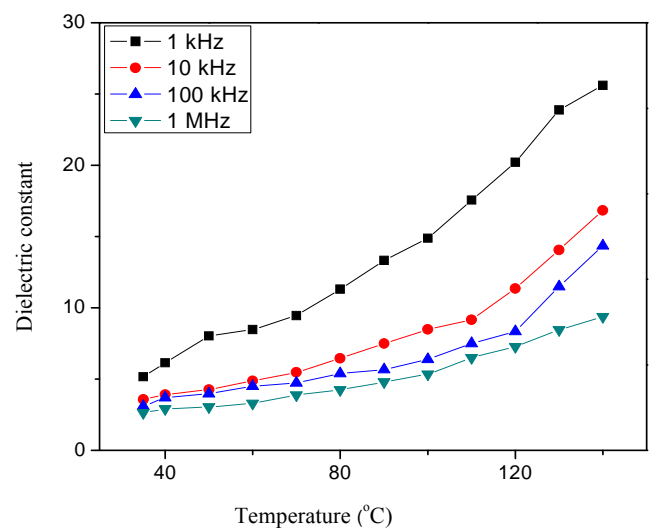

Figure 4. Temperature dependence of dielectric constant at different frequencies. 


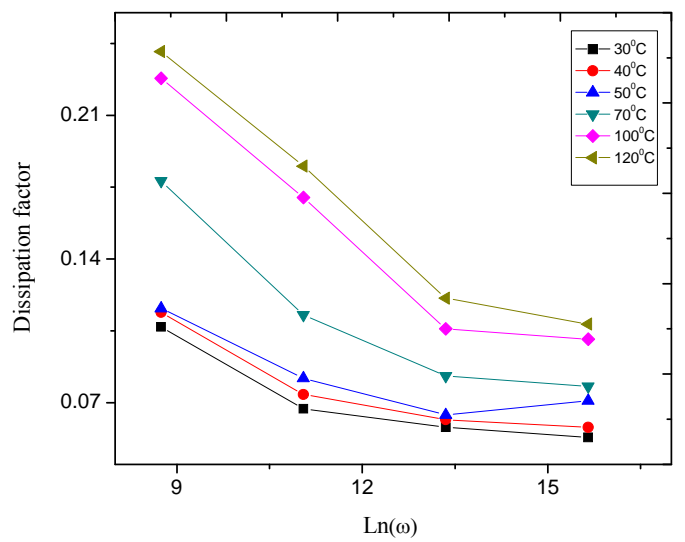

Figure 5. Frequency dependence of dissipation factor at different temperatures.

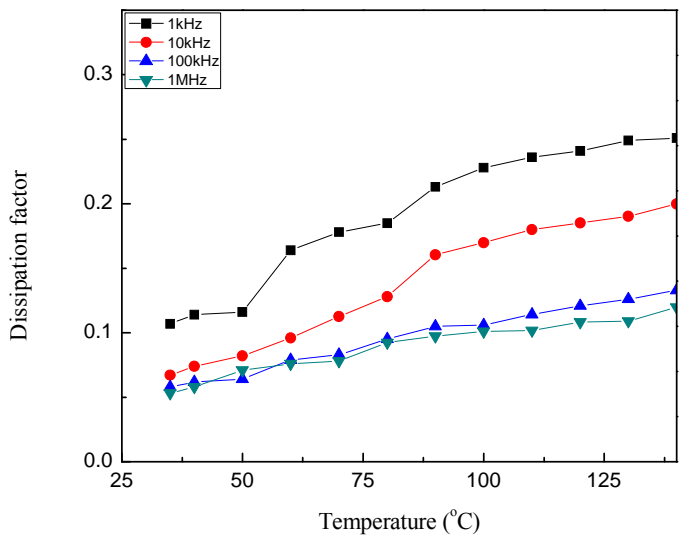

Figure 6. Temperature dependence of dissipation factor at different frequencies.

$$
\sigma_{a c}(\omega)=A \omega^{s}
$$

where $A$ is the constant, dependent on temperature and $s$ is the frequency exponent.

It is clear from Figure 7 that $\sigma_{a c}$ increases with frequency, obeying Equation (3). The values of $s$ calculated from the slopes of the plot are shown in Table 1. The frequency exponent decreases from 0.8059 to 0.7228 with increase in temperature from $35^{\circ} \mathrm{C}$ to $100^{\circ} \mathrm{C}$. It was found to be less than unity and slightly decreased with temperature. This result proposes the conduction mechanism of the grown crystals as due to Correlated Barrier Hopping $(\mathrm{CBH})$. According to this model, the hopping of carriers between two sites over a barrier separating them is responsible for the observed conductivity [10].

\section{Conclusion}

Good quality crystals of indium telluride (InTe) were grown by the Bridgman technique. The stoichiometry of the compound was confirmed by X-ray powder diffraction and chemical analysis. At a load of $25 \mathrm{~g}$, the microhardness is found to be $222.44 \mathrm{~kg} / \mathrm{mm}^{2}$, whereas at

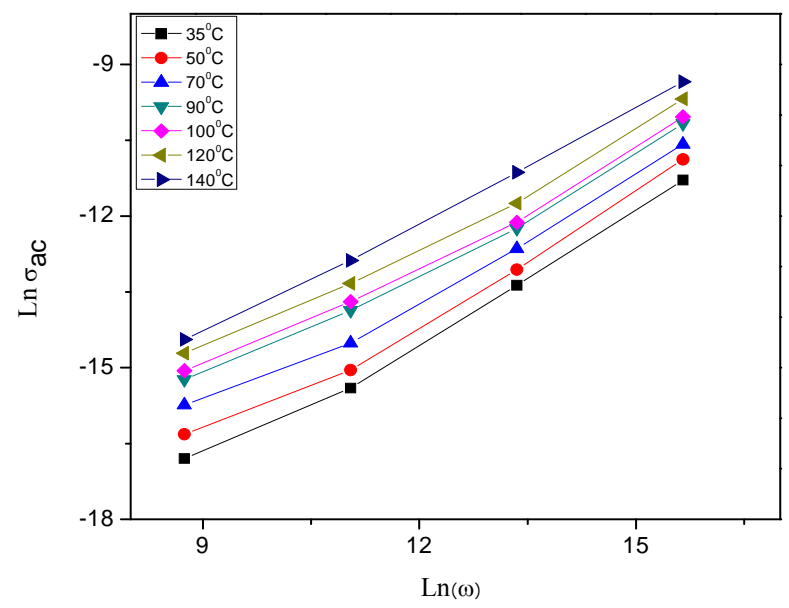

Figure 7. Logarithmic plots of AC conductivity against frequency at different temperatures.

Table 1. Values of frequency exponent at different temperatures.

\begin{tabular}{cc}
\hline Temperature $\left({ }^{\circ} \mathrm{C}\right)$ & Frequency exponent $(s)$ \\
\hline 35 & 0.8059 \\
50 & 0.7952 \\
70 & 0.7535 \\
90 & 0.7304 \\
100 & 0.7228 \\
\hline
\end{tabular}

higher loads, a decrease in hardness was observed due to slip mechanism. Further, the hardness remains constant and exhibits comparatively larger value than that of other telluride samples. The dielectric properties of the grown InTe crystals were studied for different frequencies as a function of temperature. The increase in dielectric constant as well as dielectric loss with temperature is due to the enhanced polarization of the system. The AC conductivity was observed to vary as $\omega^{s}$ in the chosen frequency range. The decrease in the value of $s$ with temperature suggests that, the $\mathrm{CBH}$ model is the predominant mechanism responsible for conduction.

\section{Acknowledgements}

The authors would like to thank the University Grants Commission, New Delhi for providing the facilities to perform microindentation analysis of the samples. Thanks are due to Prof. Guru Row, Department of Solid State and Structural Chemistry Unit, IISc, Bangalore for structural characterization.

\section{REFERENCES}

[1] O. A. Balitskii, V. P. Savchyn, P. V. Savchyn and Y. M. 
Fiyala, "Phase Formation in Surface Layers of GaTe and InTe Single Crystals during Thermal Oxidation in Air," Functional Materials, Vol. 12, No. 2, 2005, pp. 206-211.

[2] M. M. Nassary, S. A. Hussein, A. E. Belal and H. A. El-Shalkh, "Investigation of the Switching Phenomena in Indium Monotelluride Single Crystals," Physica Status Solidi (a), Vol. 145, No. 1, 1994, pp. 151-155. doi:10.1002/pssa.2211450114

[3] A. G. Kunjomana and K. A. Chandrasekharan, "Microhardness Studies of GaTe Whiskers," Crystal Research and Technology, Vol. 40, No. 8, 2005, pp. 782-785. doi:10.1002/crat.200410431

[4] G. R. Pandya, S. R. Bhavsar and P. H. Sony, "Effects of Anisotropy and Annealing on Microhardness of $\mathrm{In}_{x} \mathrm{Bi}_{2-x} \mathrm{Te}_{3}(\mathrm{x}=0.1$ to 0.5$)$ Single Crystals," Bulletin of Materials Science, Vol. 22, No. 1, 1999, pp. 15-16. doi:10.1007/BF02745669

[5] Y. S. Boyarskaya, D. Z. Grabko, M. I. Medinskaya and N. A. Palistrant, "Mechanical Properties of Pure and Doped InP Single Crystals Determined under Local Loading," Semiconductors, Vol. 31, No. 2, 1997, pp. 139-142. doi:10.1134/1.1187095

[6] D. Arivuoli, F. D. Gnanam and P. Ramasamy, "Growth and Microhardness Studies of Chalcogenides of Arsenic, Antimony and Bismuth," Journal of Materials Science Letters, Vol. 7, No. 7, 1988, pp. 711-713. doi:10.1007/BF00722076

[7] T. Chattopadhyay, R. P. Santandrea and H. G. Von Schnering, "Temperature and Pressure Dependence of the Crystal Structure of InTe: A New High Pressure Phase of InTe," Journal of Physics and Chemistry of Solids, Vol. 46, No. 3, 1985, pp. 351-356. doi:10.1016/0022-3697(85)90178-7

[8] G. K. Solanki, K. D. Patel, N. N. Gosai and B. P. Rahul, "Growth and Dielectric Properties of $\mathrm{SnSe}_{0.5} \mathrm{Te}_{0.5}$ Crys- tals," Research Journal of Chemical Sciences, Vol. 2, No. 10, 2012, pp. 43-48.

[9] A. M. Farid, H. E. Atyia and N. A. Hegab, "AC Conductivity and Dielectric Properties of $\mathrm{Sb}_{2} \mathrm{Te}_{3}$ Thin Films," Vacuum, Vol. 80, No. 4, 2005, pp. 284-294. doi:10.1016/j.vacuum.2005.05.003

[10] N. A. Hegab, M. A. Afifi, H. E. Atyia and M. I. Ismael, "AC Conductivity and Dielectric Properties of Amorphous $\mathrm{Ge}_{15} \mathrm{Se}_{60} \mathrm{X}_{25}(\mathrm{X}=$ As or $\mathrm{Sn}$ ) Films," Acta Physica Polonica A, Vol. 119, No. 3, 2001, pp. 416-423.

[11] D. N. Bose and S. De Purkayastha, "Dielectric and Photoconducting Properties of $\mathrm{Ga}_{2} \mathrm{Te}_{3}$ and $\mathrm{In}_{2} \mathrm{Te}_{3}$ Crystals," Materials Research Bulletin, Vol. 16, No. 6, 1981, pp. 635-642. doi:10.1016/0025-5408(81)90262-2

[12] S. Pal, D. N. Bose, S. Asokan and E. S. R. Gopal, "Anisotropic Properties of the Layered Semiconductor InTe," Sold State Communications, Vol. 80, No. 9, 1991, pp. 753-756. doi:10.1016/0038-1098(91)90902-8

[13] A. T. Nagat, G. A. Gamal and A. E. Belal, "Experimental Studies on the Thermoelectric Properties of InTe Single Crystals," Crystal Research and Technology, Vol. 25, No. 4, 1990, pp. 72-77. doi:10.1002/crat.2170250425

[14] V. M. Glazo and V. N. Vigdorovich, "Microhardness of Metals and Semiconductors," Consultants Bureau, New York, 1971.

[15] M. Meena and C. K. Mahadevan, "Effect of Added Impurities on the Electrical Properties of L-Arginine Acetate Single Crystals, Archives of Applied Science Research, Vol. 2, No. 6, 2010, pp. 185-199.

[16] M. A. M. Seyam, "Dielectric Relaxation in Polycrystalline Thin Films of $\mathrm{In}_{2} \mathrm{Te}_{3}$," Applied Surface Science, Vol. 181, 2001, pp. 128-138. doi:10.1016/S0169-4332(01)00378-6 